\title{
On switching $\mathscr{H}^{\infty}$ controllers for a class of linear parameter varying systems ${ }^{\hbar}$
}

\author{
Peng Yan ${ }^{\mathrm{a}, 1}$, Hitay Özbay ${ }^{\mathrm{b}, *, 1}$

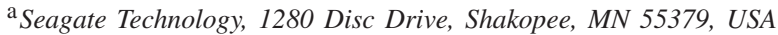 \\ ${ }^{\mathrm{b}}$ Department of Electrical and Electronics Engineering, Bilkent University, Ankara 06800, Turkey
}

Received 5 December 2005; received in revised form 14 February 2007; accepted 16 February 2007

Available online 12 April 2007

\begin{abstract}
We consider switching $\mathscr{H}^{\infty}$ controllers for a class of linear parameter varying (LPV) systems scheduled along a measurable parameter trajectory. The candidate controllers are selected from a given controller set according to the switching rules based on the scheduling variable. We provide sufficient conditions to guarantee the stability of the switching LPV systems in terms of the dwell time and the average dwell time. Our results are illustrated with an example, where switching between two robust controllers is performed for an LPV system.
\end{abstract}

(C) 2007 Elsevier B.V. All rights reserved.

Keywords: LPV systems; Asymptotic stability; Switching; Dwell time; $\mathscr{H}^{\infty}$ control

\section{Introduction}

This paper deals with switching of $\mathscr{H}^{\infty}$ controllers for a class of linear parameter varying (LPV) systems scheduled along a measurable parameter trajectory. LPV systems are ubiquitous in chemical processes, robotics systems, automative systems and many manufacturing processes. Meanwhile, Jacobian linearization of nonlinear systems also results in LPV models, where gain-scheduled controllers can be developed for the nonlinear plants. The analysis and control of LPV systems has been studied widely $[1,2,17,23,24,13,20,22]$. A systematic gain-scheduling method was developed in $[1,2]$ based on LMI (linear matrix inequality) algorithms; Grigoriadis [24] provided sufficient conditions for the stability of LPV systems with parameter-varying time delays, where gain-scheduled

\footnotetext{
is This work was supported in part by AFOSR and AFRL/VA under agreement no. F33615-01-2-3154, by the European Commission under contract no. MIRG-CT-2004-006666, and by TÜBITTAK under Grant no. EEEAG$105 \mathrm{E} 156$.

* Corresponding author. Tel.: +90312290 1449; fax: +903122664192.

E-mail addresses: Peng.Yan@seagate.com (P. Yan), hitay@bilkent.edu.tr (H. Özbay).

1 The authors were with Department of Electrical and Computer Engineering, The Ohio State University, Columbus, OH 43210, USA, during the early stages of this work.
}

controller was designed based on LMIs. Fast gain scheduling was considered in [13], where derivative information on the scheduling variable was utilized in a new control law. In a recent publication [23], an improved stability analysis for LPV systems was given and the robust gain-scheduled controller was constructed in terms of LMIs. We refer to [18] for a general review on gain-scheduling methods.

An alternative method is switching control where a family of controllers are designed at different operating points and the system performs controller switching based on the switching logic. As stated in [3], a challenging point of switching control is its hybrid nature of the continuous and discrete-valued signals. Stability analysis and the design methodology have been investigated recently in the literature of hybrid dynamical systems $[6,8,14,16,19,21]$. For LTI systems, Skafidas et al. [21] provided sufficient conditions for the stability of the switching control systems based on Filippov solutions to discontinuous differential equations and Lyapunov functionals; Morse [16] proposed a dwell time-based switching control, where a sufficiently large dwell-time can guarantee the system stability. A more flexible result was obtained in [8], where the average dwell time was introduced for switching control. Besides stability analysis, a number of results have been published on related topics, such as optimal control [19] and tracking [9]. We refer to [7] for a comprehensive review of switching 
control methods, where comparison of logic-based switching control and conventional gain-scheduling methods is provided.

Due to the time-varying and the hybrid natures of the switching LPV systems, it is challenging to explore the stability conditions and switching schemes similarly to those for LTI systems. Theoretical and practical results have been presented in recent publications $[3,11,12,14,15]$. In particular, Bett and Lemmon [3] analyzed the bounded amplitude performance and derived the conditions related to dwell time, and Lee and Lim [14] proposed switching $\mathscr{H}^{\infty}$ controllers for nonlinear systems which exhibits LPV nature after linearization. In $[11,12]$, the switched LPV systems were considered in the discrete time fashion, where the trajectory-independent LMI conditions were derived to characterize stabilization. We also notice that a quite relevant result was recently presented on switching LPV control design [15], where sufficient conditions on the hysteresis switching and the average dwell time-based switching are provided in terms of LMIs. Note that the explicit form of the dwell time and the corresponding decay rate of the switched system were not given in [15]. In the present paper, we discuss the switching $\mathscr{H}^{\infty}$ control methodology for a class of LPV systems, where each candidate $\mathscr{H}^{\infty}$ controller guarantees robust stability at the selected operating condition and the switching rules are developed to cover a large operating range. By constructing Lyapunov functionals for time-varying systems as $[4,10]$, this paper extends the stability results of $[8,16]$ to LPV systems.

The paper is organized as follows. The problem is defined in Section 2, where the structure of the candidate $\mathscr{H}^{\infty}$ controllers is described and the switching control architecture is proposed. In Section 3, the main results on the stability of the switching systems are presented in terms of the dwell time and the average dwell time. An illustrative example is given in Section 4, followed by concluding remarks in Section 5 .

\section{Problem definition}

The general structure of the switching control scheme considered in this paper is depicted in Fig. 1 , where $w_{p} \in \mathbb{R}^{n_{w}}$ is the exogenous input, $u \in \mathbb{R}^{n_{u}}$ is the control input, $z_{p} \in \mathbb{R}^{n_{z}}$ is the regulated output and $y \in \mathbb{R}^{n_{y}}$ is the measured output. The LPV system depends on a parameter $\theta(t)$, where $\theta(t) \in \mathbb{R}$ is assumed to be continuously differentiable and $\theta \in \Theta$ where $\Theta$ is a compact set.

In the present paper, we propose to construct a family of $\mathscr{H}^{\infty}$ controllers designed at selected operating points $\theta=\theta_{i}$, $i=1,2, \ldots, l$, and perform controller switching for the above LPV system, which allows for larger operating range of the LPV system. The candidate controllers are chosen from a controller set $\mathscr{K}:=\left\{K_{i}(s): i=1,2, \ldots, l\right\}$, where $K_{i}(s)$ is an LTI $\mathscr{H}^{\infty}$ controller designed for $\theta=\theta_{i}$. Consider an operating range $\Theta_{i}, \theta_{i} \in \Theta_{i}$, the LPV system in Fig. 1 can be represented as $\mathscr{F}_{u}\left(G_{\theta_{i}}, \Delta_{\theta_{i}}\right)$, where $\Delta_{\theta_{i}}$ is the time varying portion of the LPV system, $G_{\theta_{i}}$ is the LTI portion with nominal value $\theta_{i}$ and $\mathscr{F}_{u}$ denotes the upper LFT (linear fractional transformation). The closed-loop system is depicted in Fig. 2, where $G_{\theta_{i}}$ is the

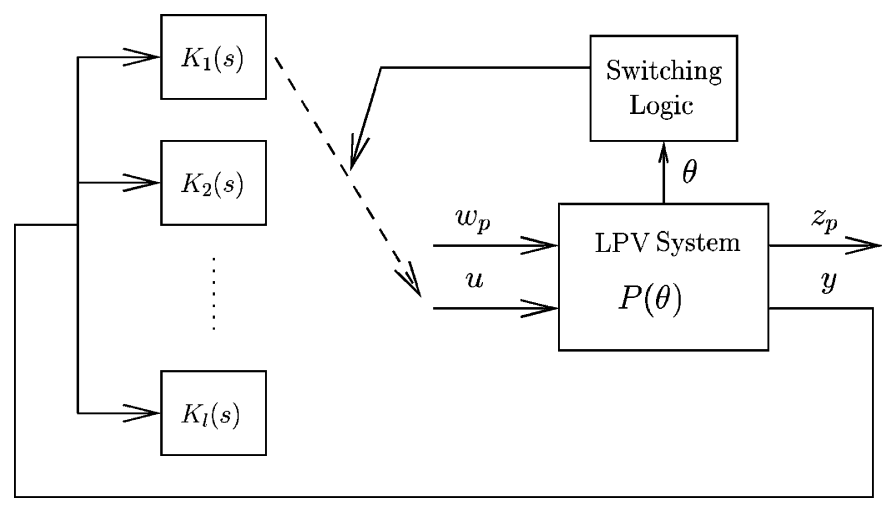

Fig. 1. The switching control system.

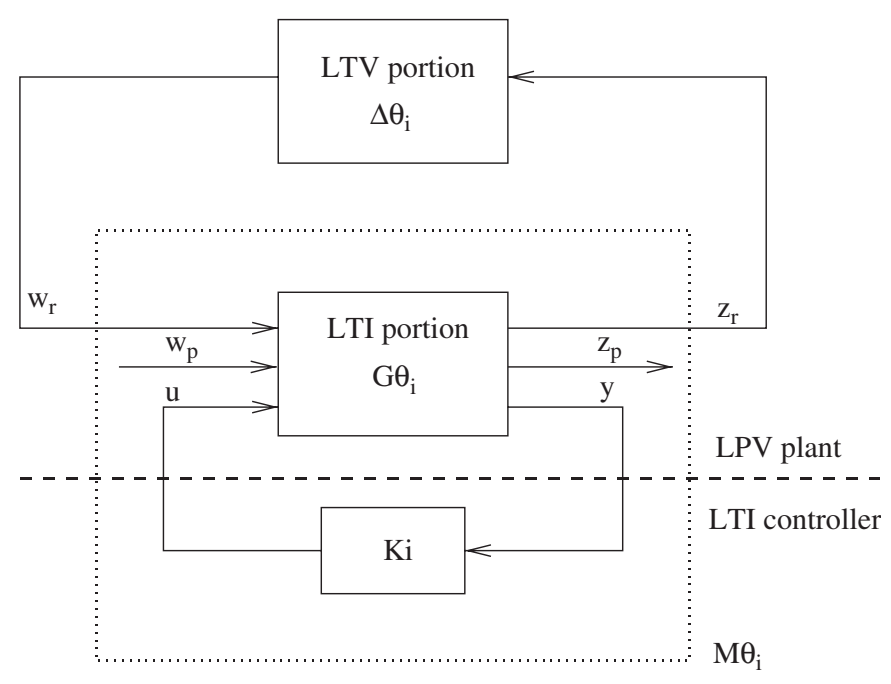

Fig. 2. LPV plant and the controller.

nominal transfer function at a specified $\theta_{i}$ :

$G_{\theta_{i}}(s)=\left[\begin{array}{c|cc}A\left(\theta_{i}\right) & B_{1}\left(\theta_{i}\right) & B_{2}\left(\theta_{i}\right) \\ \hline C_{1}\left(\theta_{i}\right) & D_{11}\left(\theta_{i}\right) & D_{12}\left(\theta_{i}\right) \\ C_{2}\left(\theta_{i}\right) & D_{21}\left(\theta_{i}\right) & D_{22}\left(\theta_{i}\right)\end{array}\right], i=1,2, \ldots l$,

and an $\mathscr{H}^{\infty}$ optimization problem is defined as finding $K_{i}(s)$ for the LTI plant $G_{\theta_{i}}$ such that (i) the closedloop system is asymptotically stable for $\theta \in \Theta_{i}$, and (ii) $\inf \left\{\sup _{w \neq 0}\left(\|z\|_{2} /\|w\|_{2}\right): K_{i}(s)\right.$ satisfies (i) $\} \leqslant \gamma$ for the smallest possible $\gamma$, where $z=\left[z_{r}^{\mathrm{T}}, z_{p}^{\mathrm{T}}\right]^{\mathrm{T}}$ and $w=\left[w_{r}^{\mathrm{T}}, w_{p}^{\mathrm{T}}\right]^{\mathrm{T}}$.

Denote $\|\cdot\|_{i, 2}$ to be the $\mathscr{L}^{2}$-induced norm and let $M_{\theta_{i}}$ to be the transfer function from $w_{r}$ to $z_{r}$. A sufficient condition on robust stability satisfying (i) is $\left\|M_{\theta_{i}}\right\|_{\infty}<1$ and $\left\|\Delta_{\theta_{i}}\right\|_{i, 2}<1$, which can be obtained by applying small gain analysis $[1,18,25]$. The above treatment results in the $\mathscr{H}^{\infty}$ controller design for the LTI system, where standard $\mathscr{H}^{\infty}$ optimization methods can be employed [5]. The state-space expression of each candidate controller $K_{i}(s)$ is given by

$K_{i}(s)=\left[\begin{array}{c|c}A_{K_{i}} & B_{K_{i}} \\ \hline C_{K_{i}} & D_{K_{i}}\end{array}\right], i=1,2, \ldots, l$. 
Note that $K_{i}(s)$ robustly stabilizes the LPV system for $\left\|\Delta_{\theta_{i}}\right\|_{i, 2}<1$, which can be guaranteed by properly choosing $\theta_{i}^{-}, \theta_{i}^{+}$and $\beta_{i}>0$, such that

$\theta \in \Theta_{i}:=\left[\theta_{i}^{-}, \theta_{i}^{+}\right], \quad|\dot{\theta}(t)|<\beta_{i}$.

In order to cover a large operating range $\Theta$, we need to develop stable switching schemes over $\mathscr{K}$. Obviously, a necessary condition for stable switching is

$\Theta \subseteq \bigcup_{i=1}^{l} \Theta_{i}$

\section{Main results}

Applying the switching rules over $\mathscr{K}$ and invoking (1) and (2), we obtain the closed loop A-matrix $A_{\mathrm{cl}} \in\left\{A_{i}(\theta), i=\right.$ $1,2, \ldots, l\}$, where

$A_{i}(\theta)=\left[\begin{array}{cc}A+B_{2} D_{K_{i}}\left(I-D_{22} D_{K_{i}}\right)^{-1} C_{2} & B_{2}\left(I-D_{K_{i}} D_{22}\right)^{-1} C_{K_{i}} \\ B_{K_{i}}\left(I-D_{22} D_{K_{i}}\right)^{-1} C_{2} & A_{K_{i}}+B_{K_{i}}\left(I-D_{22} D_{K_{i}}\right)^{-1} D_{22} C_{K_{i}}\end{array}\right]$.

For switching LTI systems, it has been shown in [16] that a sufficiently large dwell time can guarantee stability; and Hespanha and Morse [8] provided a more flexible result based on the average dwell time. We claim that similar results can be obtained for switching LPV systems.

Consider the following switching LPV system:

$\dot{\xi}(t)=A_{q}(\theta(t)) \xi(t), \quad t \geqslant 0$

where $q$ is a piecewise constant signal, which takes values on the set $\mathscr{F}:=\{1,2, \ldots, l\}$, i.e. $q(t)=i, i \in \mathscr{F}$, for $\forall t \in\left[t_{j}, t_{j+1}\right)$, where $t_{j}, j \in \mathbb{Z}^{+} \cup\{0\}$, is the $j$ th switching time instant. Here $A_{i} \in \mathscr{A}:=\left\{A_{i}(\theta(t)): i \in \mathscr{F}, \theta(t) \in \Theta\right\}$, which is a family of parameter varying matrices. We further assume that:

H1. there is a $\lambda_{i}>0$, such that for any $\theta \in \Theta$, the eigenvalues of $A_{i}(\theta)$ have real parts no greater than $-2 \lambda_{i}, \forall i \in \mathscr{F}$;

H2. $\exists K_{A}^{i}>0,\left\|A_{i}(\theta(t))\right\| \leqslant K_{A}^{i}, \forall i \in \mathscr{F}$;

H3. $\exists K_{D}^{i}>0,\left\|\frac{\partial A_{i}(\theta)}{\partial \theta}\right\| \leqslant K_{D}^{i}, \forall i \in \mathscr{F}$;

where $\|\cdot\|$ denotes the (pointwise in time) Euclidean norm of a time-varying vector and the corresponding induced norm on matrices.

The dwell time-based switching rule set is denoted by $S\left[\tau_{D}\right]$, where $\tau_{D}$ is a constant such that for any $q \in S\left[\tau_{D}\right]$, the distance between any consecutive discontinuities of $q(t), t_{j+1}-t_{j}, j \in$ $\mathbb{Z}^{+} \cup\{0\}$, is larger than $\tau_{D},[8,16]$. Clearly,

$S\left[\tau_{D 1}\right] \subset S\left[\tau_{D 2}\right], \quad \forall \tau_{D 1}>\tau_{D 2}>0$.

A sufficient condition on the minimum dwell time to guarantee the stable switching can now be given using Lyapunov stability analysis (a similar result is obtained in [14] for switched gainscheduling controllers in uncertain nonlinear systems).
First, we notice that

$\hat{A}_{i}(\theta(t)):=A_{i}(\theta(t))+\lambda_{i} I, \quad \forall i \in \mathscr{F}$

is Hurwitz, which is straightforward from (H1). Let

$Q_{i}(t)=\int_{0}^{\infty} \mathrm{e}^{\hat{A}_{i}^{\mathrm{T}}(\theta(t)) \zeta} \mathrm{e}^{\hat{A}_{i}(\theta(t)) \zeta} \mathrm{d} \zeta, \quad \forall i \in \mathscr{F}$

Note that $Q_{i}(t)$ is well defined, continuously differentiable, and is the unique positive-definite solution of

$\hat{A}_{i}^{\mathrm{T}}(\theta(t)) Q_{i}(t)+Q_{i}(t) \hat{A}_{i}(\theta(t))=-I$,

i.e.

$A_{i}^{\mathrm{T}}(\theta(t)) Q_{i}(t)+Q_{i}(t) A_{i}(\theta(t))=-2 \lambda_{i} Q_{i}(t)-I$.

Define a family of Lyapunov functions

$\mathscr{V}:=\left\{V_{i}: V_{i}(t, \xi(t)):=\xi^{\mathrm{T}}(t) Q_{i}(t) \xi(t), i \in \mathscr{F}\right\}$

for the following LPV systems, respectively:

$\dot{\xi}(t)=A_{i}(\theta(t)) \xi(t), \quad \forall i \in \mathscr{F}$.

Recall that there exist positive constants $M_{i} \geqslant \mu_{i}>0, i \in \mathscr{F}$, depending only on $\lambda_{i}$ and $K_{A}^{i}$, such that

$\mu_{i}\|\xi(t)\|^{2} \leqslant V_{i}(t, \xi(t)) \leqslant M_{i}\|\xi(t)\|^{2}, \quad t \geqslant 0$.

We refer to $[4,10]$ for details.

Consider an arbitrary switching interval $\left[t_{j}, t_{j+1}\right)$, where $q(t)=i, i \in \mathscr{F}$, for $\forall t \in\left[t_{j}, t_{j+1}\right)$. Using the quadratic form of $V_{i}$ as shown in (12), a straightforward calculation gives the time derivative of $V_{i}(t, \xi(t))$ along the trajectory of (13)

$$
\begin{aligned}
\frac{\mathrm{d}}{\mathrm{d} t} V_{i}(t, \xi(t))= & -\xi^{\mathrm{T}}(t) \xi(t)-2 \lambda_{i} \xi^{\mathrm{T}}(t) Q_{i}(t) \xi(t) \\
& +\xi^{\mathrm{T}}(t) \dot{Q}_{i}(t) \xi(t), \quad t \in\left[t_{j}, t_{j+1}\right) .
\end{aligned}
$$

Note that differentiating (9) with respect to $t$ gives

$$
\begin{aligned}
\dot{Q}_{i}(t)= & \int_{0}^{\infty} \mathrm{e}^{\hat{A}_{i}^{\mathrm{T}}(\theta(t)) \zeta}\left[\dot{\hat{A}}_{i}^{\mathrm{T}}(\theta(t)) Q_{i}(t)+Q_{i}(t) \dot{\hat{A}}_{i}(\theta(t))\right] \\
& \mathrm{e}^{\hat{A}_{i}(\theta(t)) \zeta} \mathrm{d} \zeta
\end{aligned}
$$

where

$\dot{\hat{A}}_{i}(\theta(t))=\frac{\partial}{\partial \theta} \hat{A}_{i}(\theta(t)) \dot{\theta}(t), \quad t \geqslant 0$.

Invoking (H3) and Lemma 3 of [10] we have

$\left\|\dot{\hat{A}}_{i}(\theta(t))\right\| \leqslant K_{D}^{i}|\dot{\theta}(t)|, \quad\left\|\dot{Q}_{i}(t)\right\| \leqslant K_{Q}^{i}|\dot{\theta}(t)|$,

where $K_{Q}^{i}>0$ is a constant depending only on $\lambda_{i}, K_{A}^{i}$ and $K_{D}^{i}$. Now, we are ready to state the following result. 
Theorem 3.1. Assume (H1-H3). Define

$\beta_{\max }:=\min _{i \in \mathscr{F}}\left\{\frac{1+2 \lambda_{i} \mu_{i}}{K_{Q}^{i}}\right\}$

and

$\tau_{D}=\max _{i \in \mathscr{F}}\left\{\frac{2 M_{i} \ln \sqrt{M_{i} / \mu_{i}}}{b_{i}}\right\}$,

where

$b_{i}:=\left(1+2 \lambda_{i} \mu_{i}-K_{Q}^{i} \beta\right)>0$

and $0<\beta<\beta_{\max }$. Then, the switching LPV system (6) is stable in the sense of Lyapunov for any switching rule $q \in S\left[\tau_{D}\right]$ if $|\dot{\theta}(t)| \leqslant \beta<\beta_{\max }$.

Proof. Since $|\dot{\theta}(t)| \leqslant \beta$, we have

$$
\begin{gathered}
\frac{\mathrm{d}}{\mathrm{d} t} V_{i}(t, \xi(t)) \leqslant-\left(1+2 \lambda_{i} \mu_{i}-K_{Q}^{i} \beta\right)\|\xi(t)\|^{2}=-b_{i}\|\xi(t)\|^{2}, \\
t \in\left[t_{j}, t_{j+1}\right) .
\end{gathered}
$$

Recall (14) and (22), we have

$\frac{\dot{V}_{i}(t, \xi(t))}{V_{i}(t, \xi(t))} \leqslant \frac{-b_{i}\|\xi(t)\|^{2}}{M_{i}\|\xi(t)\|^{2}}=-\frac{b_{i}}{M_{i}}$.

Thus,

$$
\|\xi(t)\| \leqslant\left\|\xi\left(t_{j}\right)\right\| \sqrt{\frac{M_{i}}{\mu_{i}}} \mathrm{e}^{\left(-b_{i} / 2 M_{i}\right)\left(t-t_{j}\right)}, \quad t \in\left[t_{j}, t_{j+1}\right) .
$$

Choosing the minimum dwell time $\tau_{D}$ obeying (20), we claim that any switching rule $q \in S\left[\tau_{D}\right]$ is stable in the sense of Lyapunov. Now, by the definition of dwell time, $t_{j+1}-t_{j}>\tau_{D}$, $j \in \mathbb{Z}^{+} \cup\{0\}$, and thus

$$
\begin{aligned}
\left\|\xi\left(t_{j+1}\right)\right\| & =\lim _{t \uparrow t_{j+1}}\|\xi(t)\| \leqslant \lim _{t \uparrow t_{j+1}}\left\|\xi\left(t_{j}\right)\right\| \sqrt{\frac{M_{i}}{\mu_{i}}} \mathrm{e}^{\left(-b_{i} / 2 M_{i}\right)\left(t-t_{j}\right)} \\
& =\left\|\xi\left(t_{j}\right)\right\| \sqrt{\frac{M_{i}}{\mu_{i}}} \mathrm{e}^{\left(-b_{i} / 2 M_{i}\right)\left(t_{j+1}-t_{j}\right)} \\
& <\left\|\xi\left(t_{j}\right)\right\| \sqrt{\frac{M_{i}}{\mu_{i}}} \mathrm{e}^{\left(-b_{i} / 2 M_{i}\right)\left(\tau_{D}\right)} \leqslant\left\|\xi\left(t_{j}\right)\right\| .
\end{aligned}
$$

Thus, we have a decreasing sequence $\left\{\left\|\xi\left(t_{j}\right)\right\|, \quad j=0,1,2, \ldots\right\}$ with upper bound $\left\|\xi\left(t_{0}\right)\right\|=\|\xi(0)\|$.

The dwell time condition in Theorem 3.1 can be applied to the switching $\mathscr{H}^{\infty}$ control problem discussed in Section 2. As depicted in Fig. 3, two possible switching schemes [14] are (a) critical-point switching and (b) hysteresis switching. For the critical-point switching, the stability of the closed-loop system cannot be guaranteed. In fact, in the worst case where $\theta(t)$ oscillates within a neighborhood of $c_{i, i+1}$, fast switching or chattering will happen, which may violate the dwell time requirement. The following corollary addresses a sufficient

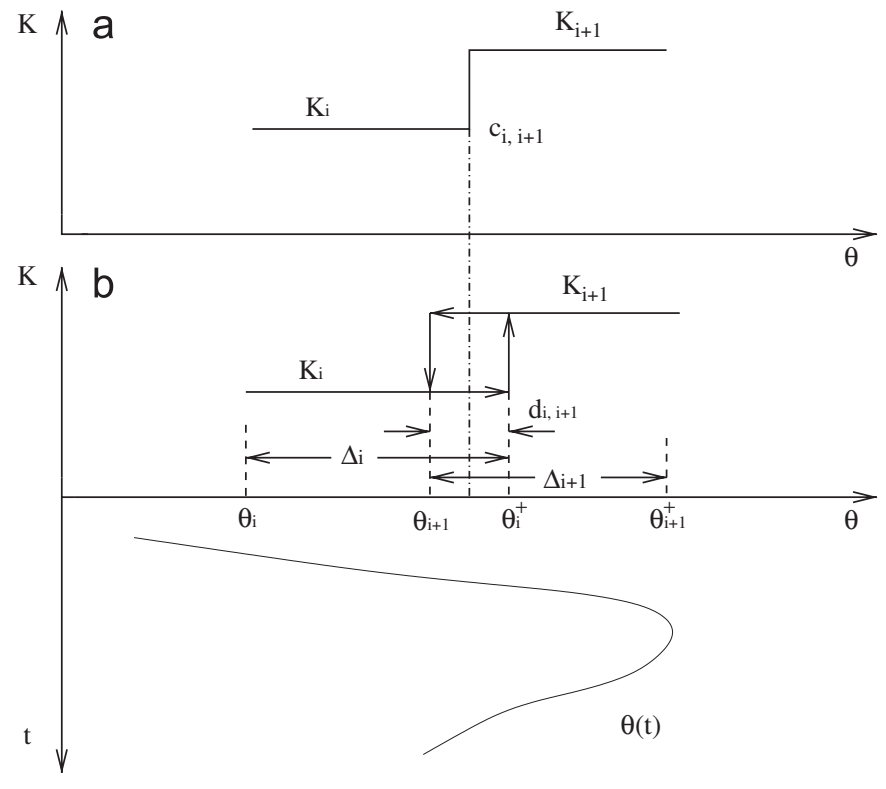

Fig. 3. Switching logic.

condition for the hysteresis switching scheme over $\mathscr{H}^{\infty}$ controller set $\mathscr{K}$.

Corollary 3.1. For the hysteresis switching over the controller set $\mathscr{K}$ with operating range $\Theta_{i}$ obeying (4), a sufficient condition for Lyapunov stability is

$|\dot{\theta}(t)|<\min \left\{\min _{i \in \mathscr{F}}\left\{\frac{\left|d_{i, i+1}\right|}{\tau_{D}}, \beta_{i}\right\}, \beta_{\max }\right\}$,

where $d_{i, i+1}=\Theta_{i} \cap \Theta_{i+1}$ is the ith hysteresis interval as shown in Fig. 3(b).

Proof. For simplicity, we consider only two neighboring controllers, i.e. $K_{i}(s)$ and $K_{i+1}(s)$ in switching time interval $\left[t_{j}, t_{j+1}\right), j \in \mathbb{Z}^{+} \cup\{0\}$. As discussed in Theorem 3.1, $t_{j+1}-t_{j}>\tau_{D}$ should be satisfied to guarantee stability of the switching system, which requires the currently working controller $K_{i}(s)$ to hold on at least $\tau_{D}$. In the worst case of switching where $\theta(t)$ oscillates around the center of the interval $d_{i, i+1}$, with amplitude $\left|d_{i, i+1}\right| / 2$, the condition $|\dot{\theta}(t)|<d_{i, i+1} / \tau_{D}$ is sufficient to guarantee stable switching. Taking all the possible controllers into consideration and invoking (3) and $|\dot{\theta}(t)|<\beta_{\max }$, we come up with (25) and complete the proof.

Note that the dwell time-based stability conditions of Theorem 3.1 and Corollary 3.1 are conservative, which do not allow for fast switching. In the following, we present another result based on the average dwell time for switching LPV systems, which can guarantee exponential stability of switching LPV systems in the more general sense.

Similar to [8], we define the average dwell time $\tau_{D}^{*}$ and the corresponding switching rule set $S_{\text {ave }}\left[\tau_{D}^{*}, N_{0}\right]$ as follows. For $t>\tau \geqslant 0$, let $N(t, \tau) \in \mathbb{Z}^{+} \cup\{0\}$ denote the number of discontinuities (switching number) of a switching signal $q$ in 
the time interval $(\tau, t) ; S_{\text {ave }}\left[\tau_{D}^{*}, N_{0}\right]$ is defined as the set of all switching rules, $q$, that satisfy

$N(t, \tau) \leqslant N_{0}+\frac{t-\tau}{\tau_{D}^{*}}$,

where $\tau_{D}^{*}$ is called the average dwell time and $N_{0}$ the chatter bound. Obviously,

$S\left[\tau_{D}^{*}\right] \subset S_{\mathrm{ave}}\left[\tau_{D}^{*}, 1\right]$.

In the rest of this section, a sufficient condition on the exponential stability is given in terms of the average dwell time, which is an extension of Theorem 2 of [8] to the switching LPV systems.

Theorem 3.2. Define $\lambda^{*}>0$ as

$\lambda^{*}=\min _{i \in \mathscr{F}}\left\{\frac{b_{i}}{2 M_{i}}\right\}$

and let

$\rho:=\max _{i \in \mathscr{F}}\left\{\sqrt{\frac{M_{i}}{\mu_{i}}}\right\}$,

where $b_{i}, M_{i}$ and $\mu_{i}$ are defined in (21) and (14), respectively. For $\forall \lambda \in\left(0, \lambda^{*}\right)$, the switching LPV system (6) is exponentially stable with decay rate no slower than $\lambda$ for all the switching rules over $S_{\text {ave }}\left[\tau_{D}^{*}, N_{0}\right]$, where

$\tau_{D}^{*}:=\frac{\ln \rho}{\lambda^{*}-\lambda}$

and $N_{0} \geqslant 0$ is any finite chatter bound.

Proof. Given time interval $\left[t_{0}, t^{\prime}\right], t^{\prime}>t_{0}=0$, denote $t_{1}<t_{2}<\cdots<t_{N\left(t^{\prime}, t_{0}\right)}$ to be the switching time instants of $q$ in $\left(t_{0}, t^{\prime}\right)$. Recall (28) and (24), we have

$$
\begin{aligned}
\left\|\xi\left(t_{1}\right)\right\| & =\lim _{t \uparrow t_{1}}\|\xi(t)\| \leqslant\left\|\xi\left(t_{0}\right)\right\| \sqrt{\frac{M_{i}}{\mu_{i}}} \mathrm{e}^{\left(-b_{i} / 2 M_{i}\right)\left(t_{1}-t_{0}\right)} \\
& \leqslant \rho\left\|\xi\left(t_{0}\right)\right\| \mathrm{e}^{-\lambda^{*}\left(t_{1}-t_{0}\right)} .
\end{aligned}
$$

Iterating the above inequality from 0 to $N\left(t^{\prime}, t_{0}\right)-1$ yields

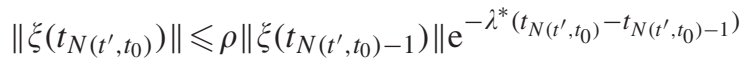

$$
\begin{aligned}
& \leqslant \cdots \leqslant \rho^{N\left(t^{\prime}, t_{0}\right)}\left\|\xi\left(t_{0}\right)\right\| \mathrm{e}^{-\lambda^{*}\left(t_{N\left(t^{\prime}, t_{0}\right)}-t_{0}\right)} .
\end{aligned}
$$

Based on (24), (31),

$$
\begin{aligned}
\left\|\xi\left(t^{\prime}\right)\right\| & \leqslant \rho^{N\left(t^{\prime}, t_{0}\right)+1}\left\|\xi\left(t_{0}\right)\right\| \mathrm{e}^{-\lambda^{*}\left(t^{\prime}-t_{0}\right)} \\
& \leqslant\left\|\xi\left(t_{0}\right)\right\| \mathrm{e}^{-\lambda^{*}\left(t^{\prime}-t_{0}\right)+\left(N\left(t^{\prime}, t_{0}\right)+1\right) \ln \rho} .
\end{aligned}
$$

For any constant $k>0$, we define

$$
N_{0}:=\frac{k}{\ln \rho}
$$

Based on the definition of $S_{\text {ave }}\left[\tau_{D}^{*}, N_{0}\right]$, we come up with

$N\left(t^{\prime}, t_{0}\right) \leqslant N_{0}+\frac{t^{\prime}-t_{0}}{\tau_{D}^{*}}$,

which is equivalent to

$-\lambda^{*}\left(t^{\prime}-t_{0}\right)+N\left(t^{\prime}, t_{0}\right) \ln \rho \leqslant k-\lambda\left(t^{\prime}-t_{0}\right)$.

Thus,

$$
\left\|\xi\left(t^{\prime}\right)\right\| \leqslant \rho\left\|\xi\left(t_{0}\right)\right\| \mathrm{e}^{-\lambda^{*}\left(t^{\prime}-t_{0}\right)+N\left(t^{\prime}, t_{0}\right) \ln \rho} \leqslant \rho\left\|\xi\left(t_{0}\right)\right\| \mathrm{e}^{k-\lambda\left(t^{\prime}-t_{0}\right)} .
$$

We conclude from (35) that the switching LPV system (6) is exponentially stable for all switching rules over $S_{\text {ave }}\left[\tau_{D}^{*}, N_{0}\right]$ with decay rate no slower than $\lambda$.

Recall (27)-(29), we have

$\tau_{D}^{*}>\tau_{D}^{*}:=\frac{\ln \rho}{\lambda^{*}}=\frac{\ln \left(\max _{i \in \mathscr{F}}\left\{\sqrt{M_{i} / \mu_{i}}\right\}\right)}{\min _{i \in \mathscr{F}}\left\{b_{i} / 2 M_{i}\right\}} \geqslant \tau_{D}$.

Thus, the average dwell time $\tau_{D}^{*}$ derived in Theorem 3.2 is larger than the minimum dwell time $\tau_{D}$ in Theorem 3.1. However, the former could allow for fast switchings because its dwell time condition is in the average sense.

Note that we assume $\theta(t)$ is a scalar function of time $t$. For the scenario $\theta(t) \in \mathbb{R}^{n_{\theta}}$ being a vector, same results can be easily obtained with similar arguments.

\section{Numerical example}

In this section, we apply the above switching $\mathscr{H}^{\infty}$ control method to the following LPV system shown in Fig. 4. We employ $\mathscr{L}\left\{\left.f(t, \theta)\right|_{\theta=\theta_{0}}\right\}=f_{\theta_{0}}(s)$ to describe the LPV dynamic equations in Laplace domain at fixed parameter values, by which the LPV plant $P_{\theta}$ can be written as

$P_{\theta}(s)=\frac{(1-\tau s)(1+\alpha s)}{(1+\tau s)\left(s^{2}+2 \xi_{0}(\theta) \omega_{0} s+\omega_{0}^{2}\right)(1-\alpha s)}$,

where $\tau=0.1, \omega_{0}=10, \alpha=15, \xi_{0}(\theta)=0.075 \theta+0.085$, and the periodic function $\theta(t)=\theta(t+T)$ is defined by

$$
\begin{aligned}
\theta(t)= & \left(3+2 \sin \left(\frac{4 \pi t}{T}\right)\right)\left(\mathbb{U}(t)-\mathbb{U}\left(t-\frac{3 T}{8}\right)\right) \mathbb{U}\left(t-\frac{7 T}{8}\right) \\
& +\mathbb{U}\left(t-\frac{3 T}{8}\right)-\mathbb{U}\left(t-\frac{7 T}{8}\right), \quad t \leqslant T,
\end{aligned}
$$

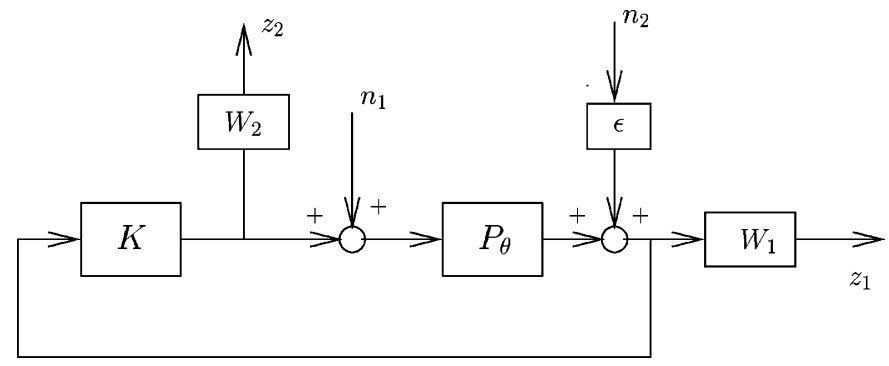

Fig. 4. Block diagram. 

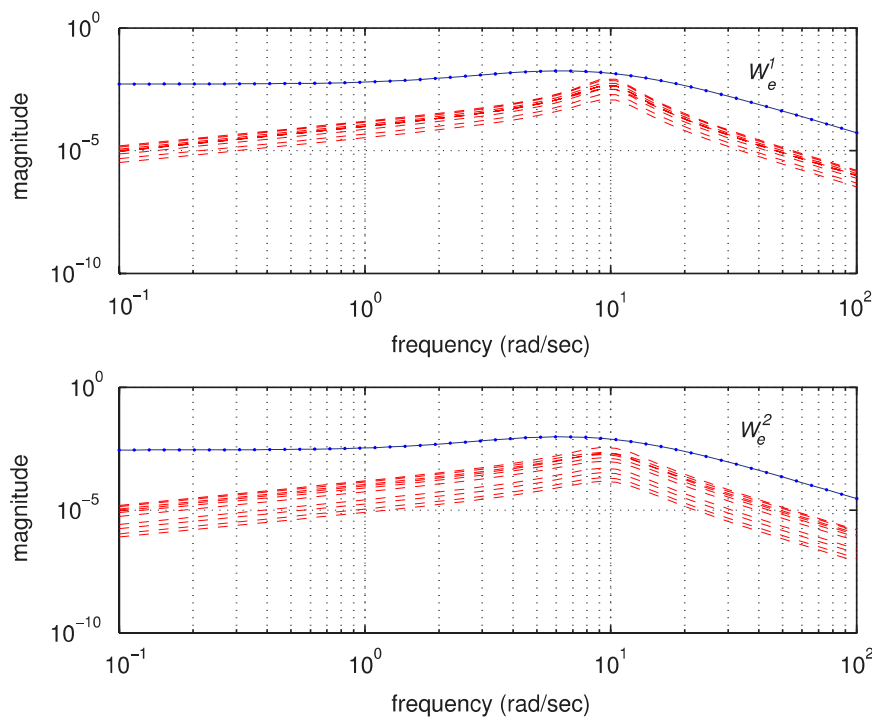

Fig. 5. Uncertainty weights.

where $T=3.6 \times 10^{4}$ and $\mathbb{U}(t)$ is the unit step function. Thus, $\theta \in \Theta:=[1,5]$ and $\xi_{0}(\theta) \in[0.16,0.46]$.

We would like to design $\mathscr{H}^{\infty}$ controllers to stabilize the system and minimize $\sup _{w \neq 0}\left\{\|z\|_{2} /\|w\|_{2}\right\}$, where the regulated output $z$ is defined as $z=\left[z_{1}, z_{2}\right]^{\mathrm{T}}$ and the exogenous input $w=\left[n_{1}, n_{2}\right]^{\mathrm{T}}$. Note that $n_{2}$ is a fictitious noise that we added so that the rank conditions of standard four block $\mathscr{H}^{\infty}$ design can be satisfied [5]. The weighting functions $W_{1}$ and $W_{2}$ are chosen as $W_{1}=(s+100) /(4 s+4)$ and $W_{2}=2$, respectively.

We design two $\mathscr{H}^{\infty}$ controllers $K_{1}$ and $K_{2}$ at the operating points $\theta=\theta_{1}=2.2$ and $\theta=\theta_{2}=3.8$, respectively, and employ controller switching between $K_{1}$ and $K_{2}$. The operating range is chosen as $\Theta_{1}=\left[\theta_{1}^{-}, \theta_{1}^{+}\right]=[1,3.4]$ for controller $K_{1}$, and $\Theta_{2}=$ $\left[\theta_{2}^{-}, \theta_{2}^{+}\right]=[2.6,5]$ for $K_{2}$. The two candidate $\mathscr{H}^{\infty}$ controllers $K_{1}$ and $K_{2}$ can be constructed using standard $\mathscr{H}^{\infty}$ optimization methods [5,25]:

$K_{1}(s)=\frac{138(s+2.48+j 9.70)(s+2.48-j 9.70)(s+10)}{(s+0.07)(s+8.74+j 17.83)(s+8.74-j 17.83)(1+s / 70)}$

and

$K_{2}(s)=\frac{138(s+3.72+j 9.27)(s+3.72-j 9.27)(s+10)}{(s+0.07)(s+9.37+j 17.89)(s+9.37-j 17.89)(1+s / 70)}$.

Define $P_{e}^{i}(s)=P_{\theta}(s)-P_{\theta_{i}}(s), i=1,2$, and assume

$\left|P_{e}^{i}(j \omega)\right|<\left|W_{e}^{i}\right|, \quad i=1,2$.

A sufficient condition to guarantee robust stability is given by [25]

$\left\|W_{e}^{i} K_{i}\left(1+P_{\theta_{i}} K_{i}\right)^{-1}\right\|_{\infty} \leqslant 1, \quad i=1,2$.

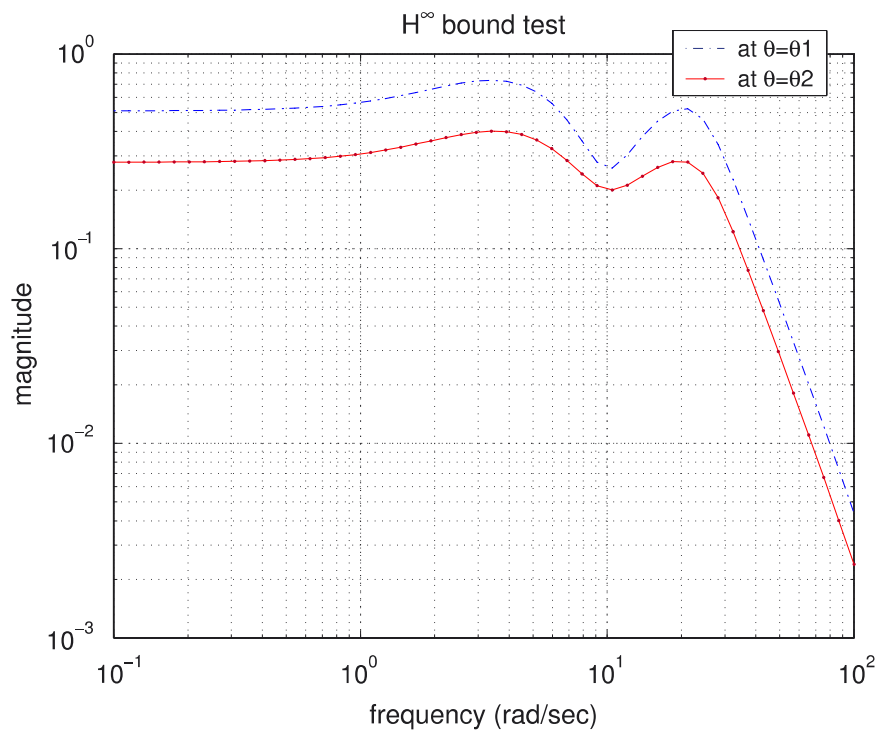

Fig. 6. Robustness test.
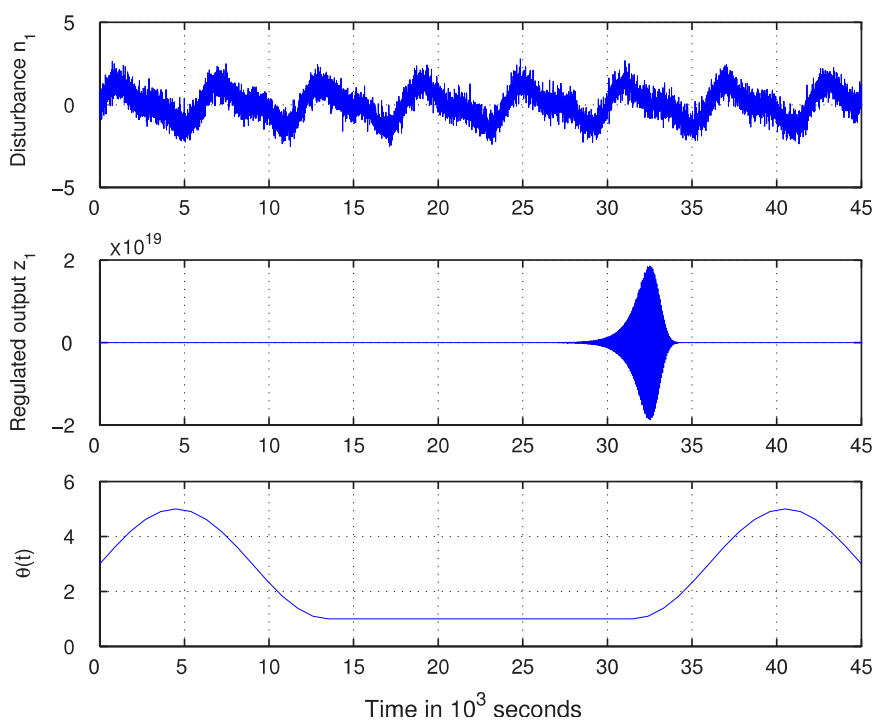

Fig. 7. The case of a single $\mathscr{H}^{\infty}$ controller.

As depicted in Fig. 5, (40) can be satisfied by choosing

$$
\begin{gathered}
W_{e}^{1}(s)=\frac{55(s+2)^{2}}{(s+7)^{2}(s+8)(s+9)(s+12)} \\
W_{e}^{2}(s)=\frac{30}{55} W_{e}^{1}(s) .
\end{gathered}
$$

Fig. 6 shows that the robust stability condition (41) is satisfied for $K_{1}$ and $K_{2}$, respectively. Thus, $K_{1}$ and $K_{2}$ can robustly stabilize the LPV system with respect to $\Theta_{1}$ and $\Theta_{2}$.

Numerically, we have $\beta_{\max }=8.6 \times 10^{-4}$ from (19). Also notice that

$|\dot{\theta}(t)| \leqslant\left|\frac{4 \pi}{18000}\right| \approx 7 \times 10^{-4}$. 

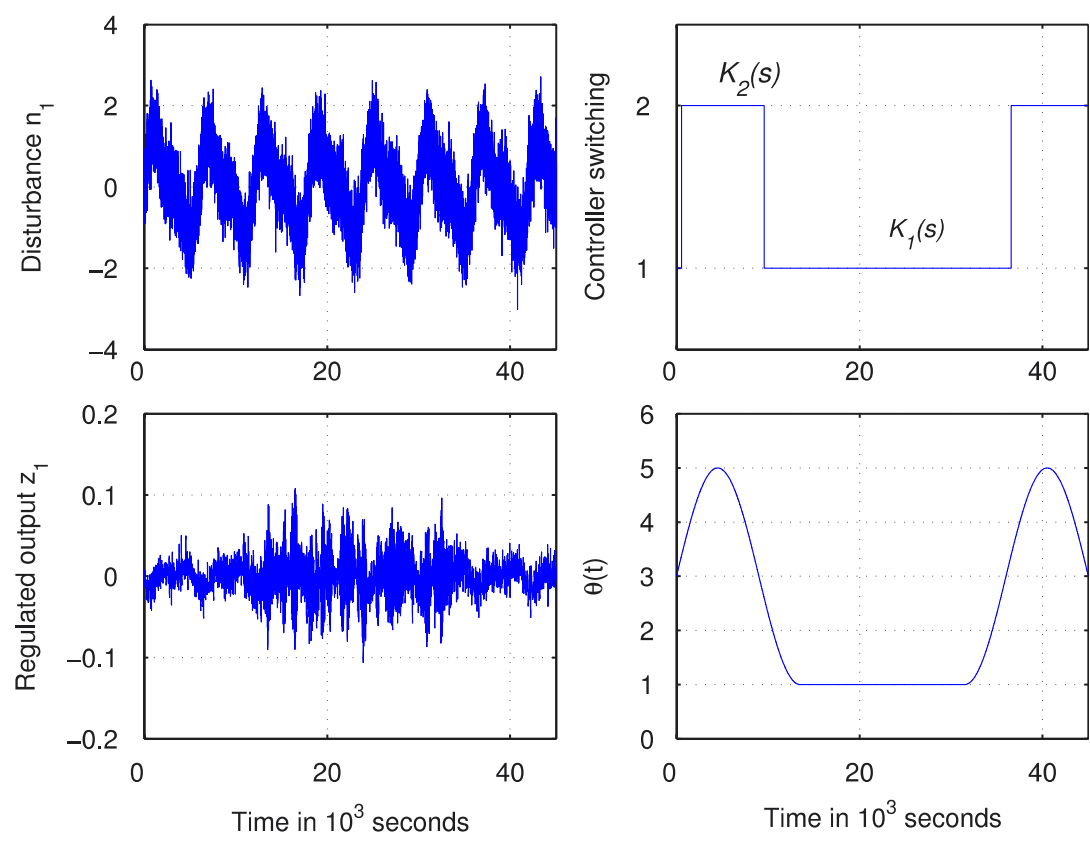

Fig. 8. The swtiching $\mathscr{H}^{\infty}$ control method.

Choosing $\beta=8.1 \times 10^{-4}<\beta_{\max }$ and invoking (21), we have $b_{1}=0.15$ and $b_{2}=2.84$. Furthermore, we can pick $\lambda=1.02 \times$ $10^{-4}$ such that $\tau_{D}^{*}=1.5 \times 10^{4}$, which is straightforward from (27)-(29). Thus, the switching scheme for $\theta(t)$ belongs to $S_{\text {ave }}\left[\tau_{D}^{*}, 1\right]$, which is due to the fact that there are only two switchings per period $T$ (Fig. 8). Based on Theorem 3.2, we conclude that the switching LPV system with $K_{1}$ and $K_{2}$ are stable.

The closed-loop system with the determined switching $\mathscr{H}^{\infty}$ control scheme is simulated using MATLAB. For the purpose of comparison, we also provide an $\mathscr{H}^{\infty}$ controller $K_{0}$ designed for $\theta=\left(\theta_{1}^{-}+\theta_{2}^{+}\right) / 2=3$, by which the performance of a single $\mathscr{H}^{\infty}$ controller can be simulated. The disturbance $n_{1}$ is set to be $n_{1}(t)=\sin (2 \pi t / 6000)+\frac{1}{2} \sin (2 \pi t / 3000)+\delta(t)$, where $\delta(t)$ is a Gaussian distributed signal of mean 0 and variance 0.2 .

First, we give the simulation result for the case of single $\mathscr{H}^{\infty}$ controller $K_{0}$ (for comparison purposes) in Fig. 7, where the divergence of the output signal is observed because $K_{0}$ itself cannot robustly stabilize the LPV system for the whole operating range $\Theta$. Simulations of the switching $\mathscr{H}^{\infty}$ control method are depicted in Fig. 8. Note that the system remains stable and the magnitude of the regulated output $z_{1}$ is much smaller than the magnitude of the disturbance $n_{1}$ for all $\theta \in \Theta$.

Note that for the proposed switching control scheme, conditions of Theorem 3.1 are not satisfied: the minimum dwell time $\tau_{D}$ to guarantee stability in Theorem 3.1 is given by

$\tau_{D}=\max _{i=1,2}\left\{\frac{2 M_{i} \ln \sqrt{M_{i} / \mu_{i}}}{b_{i}}\right\}=9.71 \times 10^{3}>\tau_{\min }$,

where $\tau_{\min }=T / 4=9000$ is the minimum distance between two consecutive switchings in our design, which is depicted in Fig. 8. Meanwhile, Corollary 3.1 also turns out to be too conservative for this design due to the fact that

$\max \{\dot{\theta}(t)\}=\frac{4 \pi}{18000}>\frac{\left|d_{1,2}\right|}{\tau_{D}}=\frac{0.8}{9710}=8.2 \times 10^{-5}$,

which violates (25). The analysis of this numerical example affirms a good coincidence with the discussion of Section 3. It suggests that Theorem 3.2 is a less conservative result allowing faster switching.

\section{Concluding remarks}

Switching $\mathscr{H}^{\infty}$ controllers are proposed for a class of LPV systems with slow parameter variations. Controller robustness is combined with the switching policy, which results in the hysteresis switching over a set of $\mathscr{H}^{\infty}$ controllers designed at selected operating points. The stability analysis is provided in terms of the dwell time and the average dwell time. The proposed switching $\mathscr{H}^{\infty}$ control method is illustrated by a numerical example, where the comparison between the single $\mathscr{H}^{\infty}$ controller and our design is also given. A further extension of this work would be switching control for LPV systems with fast parameter variations.

\section{References}

[1] P. Apkarian, P. Gahinet, A convex characterization of gain-scheduled $\mathscr{H}^{\infty}$ controllers, IEEE Trans. Automat. Control 40 (1995) 853-864.

[2] P. Apkarian, P. Gahinet, G. Becker, Self-scheduled $\mathscr{H}^{\infty}$ control of linear parameter-varying systems: a design example, Automatica 31 (1995) 1251-1261.

[3] C. Bett, M. Lemmon, Bounded amplitude performance of switched LPV systems with applications to hybrid systems, Automatica 35 (1999) 491-503.

[4] C. Desoer, Slowly varying system $\dot{x}=A(t) x$, IEEE Trans. Automat. Control 14 (1969) 780-781. 
[5] J. Doyle, K. Glover, P. Khargonekar, B. Francis, State-space solutions to standard $\mathscr{H}_{2}$ and $\mathscr{H}_{\infty}$ control problems, IEEE Trans. Automat. Control 34 (1989) 831-847.

[6] J. Hespanha, Uniform stability of switched linear systems: extension of Lasalle's invariance principle, IEEE Trans. Automat. Control 49 (2004) 470-482.

[7] J. Hespanha, D. Liberzon, S. Morse, Overcoming the limitations of adaptive control by means of logic-based switchin, Syst. Control Lett. 49 (2003) 49-65.

[8] J. Hespanha, S. Morse, Stability of switched systems with average dwelltime, Proceedings of the 38th IEEE Conference on Decision and Control, Phoenix, December 1999, 2655-2660.

[9] J. Hochcerman-Frommer, S. Kulkarni, P. Ramadge, Controller switching based on output prediction errors, IEEE Trans. Automat. Control 43 (1998) 596-607.

[10] D. Lawrence, W. Rugh, On a stability theorem for nonlinear systems with slowly varying inputs, IEEE Trans. Automat. Control 35 (1990) 860-864.

[11] J.-W. Lee, On uniform stabilization of discrete-time linear parametervarying control systems, IEEE Trans. Automat. Control 51 (2006) 1714-1721.

[12] J.-W. Lee, G. Dullerud, Uniform stabilization of discrete-time switched and Markovian jump linear systems, Automatica 42 (2006) 205-218.

[13] S.-H. Lee, J.-T. Lim, Fast gain scheduling on tracking problems using derivative information, Automatica 33 (1997) 2265-2268.
[14] S.-H. Lee, J.T. Lim, Switching control of $\mathscr{H}^{\infty}$ gain scheduled controllers in uncertain nonlinear systems, Automatica 36 (2000) 1067-1074.

[15] B. Lu, F. Wu, Switching LPV control designs using multiple parameterdependent Lyapunov fucntions, Automatica 40 (2004) 1973-1980.

[16] S. Morse, Supervisory control of families of linear set-point controllers: part 1: exact matching, IEEE Trans. Automat. Control 41 (1996) 1413-1431.

[17] A. Packard, Gain-scheduling via linear fractional transformations, Syst. Control Lett. 22 (1994) 79-92.

[18] W. Rugh, J. Shamma, Research on gain scheduling, Automatica 36 (2000) 1401-1425.

[19] A. Savkin, E. Sakfidas, R. Evans, Robust output feedback stabilizability via controller switching, Syst. Control Lett. 29 (1996) 81-90.

[20] J. Shamma, M. Athans, Guaranteed properties of gain scheduled control for linear parameter-varying plants, Automatica 27 (1991) 559-564.

[21] E. Skafidas, R. Evans, A. Savkin, I. Peterson, Stability results for switched controller systems, Automatica 35 (1999) 553-564.

[22] D. Stilwell, W. Rugh, Stability and $\mathscr{L}_{2}$ gain properties of LPV systems, Automatica 38 (2002) 1601-1606.

[23] F. Wang, V. Balakrishnan, Improved stability analysis and gain-scheduled controller synthesis for parameter-dependent systems, IEEE Trans. Automat. Control 47 (2002) 720-734.

[24] F. Wu, K. Grigoriadis, LPV systems with parameter-varying time delays: analysis and control, Automatica 37 (2001) 221-229.

[25] K. Zhou, J. Doyle, K. Glover, Robust and Optimal Control, PrenticeHall, Englewood Cliffs, NJ, 1996. 